\title{
El Pacto Mundial para la Migración Segura, Ordenada y Regular: ¿hacia dónde vamos?'
}

\author{
Global Compact for Safe, Orderly and Regular Migration: \\ where do we go from here?
}

Jorge Martínez Pizarro*

ISSN IMPRESO 1870-7599 | ISSN RED CÓMPUTO 2448-7783 | 25-40

RECIBIDO 25/08/20 | ACEPTADO 28/10/20

\begin{abstract}
Resumen. Se examina y describe de manera general el Pacto Mundial para una Migración Segura, Ordenada y Regular; además, se hace alusión a sus antecedentes, alcances, objetivos y contenidos. Este instrumento de soft law de las Naciones Unidas es otra iniciativa más de un proceso de afirmación progresiva de la migración en la escala global. A pesar de los pocos análisis a que ha dado lugar a la fecha, así como la indefinición de la tríada que plantea, la falta de especificaciones de sus propuestas de acción y la incipiente implementación en los países después de su ratificación en 2018, nada parece interponerse para prestarle atención a sus posibilidades, propuestas y definiciones acerca de la gobernanza de la migración contemporánea.
\end{abstract}

Palabras clave: pacto, gobernanza de la migración, América Latina y el Caribe, derechos humanos, desarrollo.

Abstract. This article provides an overview of the Global Compact for Safe, Orderly and Regular Migration; further, it refers to the agreement's antecedents, reach, objectives and contents. This soft law instrument of the United Nations is one more advance toward a global progressive statement on migration. Although there have been little analysis of the Pact to date, including of the uncertain definition of the triad of actions that it proposes, the lack of specifics in its proposals for action and the tentative implementation by countries following its ratification in 2018, nothing appears to stand in the way of its possible outcomes, proposals and definitions around governance of contemporary migration.

Keywords: compact, migration governance, Latin America and the Caribbean, human rights, development.

${ }^{1}$ Una versión previa fue presentada en el Seminario Internacional Inclusión y Cohesión Social en el marco de la Agenda 2030 para el Desarrollo Sostenible: Claves para un Desarrollo Social Inclusivo en América Latina, realizado en la sede de la Comisión Económica para América Latina y el Caribe (Cepal), en Santiago, los días 28 y 29 de mayo de 2019.

* Chileno. Investigador del Centro Latinoamericano y Caribeño de Demografía (Celade), División de Población de la Cepal, en Santiago de Chile. Correo-e: jorge.martinez@cepal.org 


\section{Introducción}

El Pacto Mundial para una Migración Segura, Ordenada y Regular, descrito en este trabajo, es una iniciativa de las Naciones Unidas destinada a fijar nociones, enfoques y acciones para la gobernanza de la migración contemporánea. Por tal razón, debe entenderse como un instrumento de largo aliento cuya implementación deberá evaluarse periódicamente. El Pacto tiene varias características que serán analizadas a continuación; varias de ellas pueden ser vistas como fortalezas, en tanto que otras constituyen debilidades indiscutibles que seguramente se irán enfrentando. En principio, hay que destacar que es un instrumento con propuestas integrales y abarcadoras para la acción basadas en la protección de los derechos humanos, lo que promueve una actitud claramente afirmativa de los procesos migratorios. Pero, al mismo tiempo, la tríada «segura, ordenada y regular», que lleva un buen tiempo en la jerga de la "diplomacia migratoria» parece estar distante de teorías, conceptos, enfoques y debates en torno de la migración, lo que exige conocer su significado, en este caso, en los contenidos del Pacto.

Ahora bien, a pesar de vincularse con la Agenda 2030 de Desarrollo Sostenible, los objetivos y líneas de acción del Pacto carecen de definiciones claras en cuanto a indicadores, fuentes, metadatos, horizontes y metas. Este es un tema que cobra relevancia para toda política que busque, efectivamente, hacer de la migración un proceso ordenado, seguro y regular. El riesgo de apropiación de la tríada, en ausencia de definiciones claras con las condiciones señaladas, puede vaciar de contenidos su propia denominación. No se discute aquí el carácter no vinculante del Pacto: ello es más una fortaleza que una debilidad, pues los Estados que lo han ratificado no se ven obligados a adoptar normativas y ajustes a sus ordenamientos constitucionales, tareas que impedirían su implementación.

\section{Una iniciativa de soft law}

En diciembre de 2018 el Pacto Mundial para una Migración Segura, Ordenada y Regular fue ratificado por 164 Estados miembros de las Naciones Unidas, luego de un año de consultas regionales de amplia convocatoria que confluyeron en una reunión de carácter global (2017), y otro año de negociaciones entre los 
Estados, en el que se fue dando forma a sus contenidos y objetivos (2018). Durante 2019 y 2020 se esperaba que los países comenzaran su implementación y brindaran informes voluntariamente a fines de 2020.

El Pacto es un instrumento de la llamada soft law y constituye la cristalización de múltiples iniciativas que han tenido lugar durante varias décadas en el ámbito de las Naciones Unidas, las que se remontan al menos a la aprobación de la Convención Internacional para los Trabajadores Migrantes y sus Familias en 1990, que entró en vigor 13 años después, y al Programa de Acción de El Cairo aprobado en la Conferencia Internacional sobre Población y Desarrollo celebrada en 1994. Al respecto, la necesidad de este instrumento puede ser vista, de entrada, desde dos perspectivas:

a) Se trata de una muestra del fracaso de dichas iniciativas globales, que no lograron concretar a cabalidad los planteamientos originales acerca de la gobernanza migratoria global, pese a los muchos recursos invertidos en la sensibilización de las problemáticas que afectan a las personas migrantes.

b) El Pacto en cuestión es un estadio esperado en la lucha de organizaciones internacionales, sociedad civil, migrantes y academia, sobre el cual cabe apoyar su implementación en cada país, habida cuenta de sus preceptos de favorecer una participación amplia y sin condiciones de diversos actores, sujeta a compromisos, voluntades y recursos.

Como quiera que sea, puede decirse, por lo tanto, que existe en el mundo y en América Latina una arquitectura monumental a favor de la migración internacional y la protección de los migrantes, no en su contra (Martínez, 2017), si bien la realidad es contrastante, ya que numerosas personas migrantes y familias enteras enfrentan de forma cotidiana situaciones de riesgo, fobias y discriminaciones de todo tipo, las cuales pareciera que se han exacerbado en el contexto de la pandemia durante 2020, mientras que persisten o se refuerzan visiones centradas en el control migratorio, en lo que constituye una de las tensiones globales más preocupantes en la actualidad.

\section{El Pacto en su contexto}

Son escasas las descripciones, exámenes, cuestionamientos y propuestas acerca de este Pacto desde su puesta en ejecución, lo que hace difícil confrontar a sus defensores con sus detractores, más allá de los países que no lo ratificaron. 
Durante 2020 los países debían preparar su primer informe voluntario de cara a una revisión regional de implementación, lo que se pospuso, al menos en la región, para inicios de 2021. La retórica dominó las alusiones públicas en el primer semestre de 2019, lo que puede clasificarse en las siguientes posturas:

a) Quienes, desde su formulación, han manifestado que se trata de una hoja de ruta para abordar los desafíos de la migración de cara al siglo XXI.

b) Quienes abiertamente lo desprecian por considerarlo un instrumento disuasivo y problematizador de la migración, elaborado con terminologías del Norte global al servicio de sus intereses.

c) Quienes lo califican («acusan») de constituir un pacto a favor de la migración y en consecuencia lo responsabilizan de fomentar la migración irregular y la violación de la soberanía nacional.

En primer lugar, debe reiterarse el carácter no vinculante del Pacto, atributo que puede ser descrito como una fortaleza porque proporciona una flexibilidad respecto de qué acciones y qué objetivos pueden ser implementados según el interés de cada país. Las iniciativas de esa naturaleza impulsan acuerdos y cierto tipo de compromisos entre los países, promueven la participación de la sociedad civil y son objeto de exámenes críticos reformuladores de sus contenidos. En segundo lugar, hay que destacar que los contenidos del Pacto se vinculan con un proceso que guarda directa relación con la resolución del Segundo Diálogo de Alto Nivel sobre Migración y Desarrollo de 2013 (Martínez, 2015), con la Agenda 2030 de Desarrollo Sostenible (United Nations, 2015) y, especialmente, con la Declaración de Nueva York sobre Migrantes y Refugiados del año 2016. En esta última es que los países se comprometen, en la Asamblea General de las Naciones Unidas, a elaborar dos pactos, uno sobre refugiados y otro sobre migración (ONU, 2016). A pesar de que la división puede no ser clara en la actualidad, existen entonces dos pactos diferenciados en razón de sus propósitos y poblaciones objetivo.

Por varias razones en América Latina se ha dado un uso conjunto cada vez más habitual de los términos «migrantes y refugiados», lo que habrá que evaluar con el tiempo. La distinción puede volverse difusa si se reconoce el crecimiento de las migraciones mixtas, como lo ilustra el escenario en el norte de Centroamérica, en Haití y en la migración venezolana. Pero es claro que las definiciones del estatus de refugiado y las de migrante no son asimilables en el derecho internacional. ${ }^{2}$

${ }^{2}$ El Pacto Mundial sobre los Refugiados tampoco es vinculante. Sus objetivos son: a) aliviar las presiones sobre los países de acogida, b) promover la autosuficiencia de los refugiados, c) ampliar el acceso 
En tercer lugar, el proceso del Pacto muestra que, por un lado, hay una manifiesta preocupación por la situación migratoria vigente, que intenta reflejar una posición objetiva centrada en la necesidad de la protección de las personas migrantes, pero en la que se da cabida a diversos intereses en juego representados esencialmente por las temáticas de la soberanía. Esto es lo que explica el contenido político de la «oferta» del Pacto para entregar elementos destinados a la gobernanza de las migraciones contemporáneas, en un contexto que busca garantizar una migración segura, ordenada y regular. Como la gobernanza es un tema altamente complejo (cabría preguntarse qué lugar ocupan las remesas en ella) y que no sólo incluye la cooperación internacional y los acuerdos de todo tipo, es claro que los intereses en juego confrontan un énfasis diferente según si la mirada de la situación vigente proviene del Norte o del Sur global e, incluso, dentro de este último.

Se puede decir, como una primera aproximación de balance, que el Pacto da cuenta de una plena transición de gobernanza migratoria, la que no se desarrollará en el corto plazo sino que probablemente tomará un largo tiempo y desplegará su implementación en las escalas regionales y globales en medio de tensiones como las indicadas. En esto se requiere identificar objetivos urgentes de lograr y otros que deberán implementarse progresivamente.

¿Segura, ordenada y regular?

Sin duda, desde el punto de vista académico, para muchos observadores y para la sociedad civil, la tríada segura, ordenada y regular es un injerto extraño, alejado de conceptos propios del campo de la investigación en torno de la migración, y resulta hasta un llamado a la obediencia. Es, en el fondo, una incitación a la crítica a los modelos de gobernanza migratoria.

Es importante destacar que hay abono para quienes han hecho cuestionamientos a esta iniciativa, señalando que el Pacto y su tríada harían ver que la migración es en gran medida irregular, por lo cual es un problema que hay que controlar, lo que justifica las restricciones y el control (Ramírez y otros, 2019), así

a las soluciones que impliquen a terceros países, y d) favorecer en los países de origen condiciones que propicien un retorno en condiciones de seguridad y dignidad. El pacto mundial tratará de lograr esos cuatro objetivos interrelacionados e interdependientes mediante la movilización de la voluntad política, la ampliación de la base de apoyo y acuerdos entre los Estados y otras partes interesadas pertinentes con miras a facilitar contribuciones más equitativas, sostenidas y previsibles (ONU, 2018). 
como las detenciones, deportaciones y con ello los discursos promotores y reproductores de fobias, discriminaciones y violaciones a los derechos humanos.

La cuestión de la migración segura, ordenada y regular está lejos de ser conceptualmente articulada y asimilable a algún cuerpo teórico de las migraciones. En realidad, no se ha definido suficientemente, a pesar de que en los 23 objetivos del Pacto se pueden encontrar varias acepciones que le dan sentido y en la Agenda 2030, como en otros instrumentos precedentes, se alude a dichos términos en conjunto.

En América Latina y el Caribe hay tres países que no forman parte del Pacto (Brasil, Chile y República Dominicana), aunque sus autoridades afirman con frecuencia que les interesa que un proceso migratorio sea seguro, ordenado y regular, y han aceptado además las metas de migración en los Objetivos de Desarrollo Sostenible (ODS). Tal contradicción podría indicar la transición de gobernanza migratoria por la que se está atravesando a escala global.

Es necesario insistir en que el Pacto, a pesar de considerarse un hito histórico, no es un instrumento vinculante. Es más bien un marco de acción basado en la Declaración de Nueva York, que parte por recordar dos premisas antiguas: por un lado, que no hay ningún Estado que pueda asumir por sí solo los desafíos asociados a los refugiados y a la migración; y, por otro, que los Estados han ido asumiendo compromisos y obligaciones respecto al derecho internacional en lo que concierne a su propia sociedad.

En las reuniones previas a la firma del Pacto mundial varios Estados reconocieron que éste es un instrumento que no tiene precedentes en el ámbito de procesos sociales globales, como es el caso de la migración. Se consideró a la migración como un asunto positivo, permanentemente amenazado por distintas fuerzas, entre ellas, la poderosa impronta de la falta de conocimiento. De ahí que el tema de la evidencia (la falta de ella) apareciera en reiteradas ocasiones durante las negociaciones. Para algunos países la evidencia mostraba lo inaceptable que es tener personas en situación irregular, lo preocupante que es el retorno de la apatridia, mientras que para otros confirmaba la necesidad de conocer tendencias de flujos de migrantes en distintas regiones $y$, sobre todo, en su relación con el desarrollo sostenible. 


\section{Declaración de Nueva York para los Refugiados y los Migrantes y Agenda 2030}

La Declaración de Nueva York para los Refugiados y los Migrantes, adoptada el 19 de septiembre de 2016 durante la Primera Reunión de Alto Nivel de la Asamblea General sobre la respuesta a los grandes desplazamientos de refugiados y migrantes, es el instrumento político central del proceso de creación de los dos pactos. La declaración estableció una serie de compromisos de los Estados miembros de las Naciones Unidas en materia de migración internacional y refugio. Aun cuando no es vinculante para los países adherentes, debido a su carácter de declaración, representa evidentemente la instalación de la necesidad de avances en la normativa internacional para garantizar los derechos de los migrantes y profundizar las metas de migración internacional abordadas en la Agenda 2030.

En la sección de compromisos, tanto con los refugiados como con los migrantes, en el párrafo 22, los países se comprometen a adoptar un enfoque integral centrado en las personas y utilizando una perspectiva de género, con el propósito de garantizar los derechos humanos de los migrantes (ONU, 2016:6). En el párrafo 55, la Declaración hace referencia a los resultados de los dos primeros Diálogos de Alto Nivel sobre la Migración Internacional y el Desarrollo, celebrados en 2013 y 2017, y los reconoce como importantes instancias de diálogos. Asimismo, se menciona directamente la creación de un pacto mundial para la migración segura, ordenada y regular y se indica que la Declaración del Diálogo de Alto Nivel sobre la Migración Internacional y el Desarrollo será la base para el pacto de gobernanza migratoria (ONU, 2016:26).

Concerniente a los precedentes más directos y en línea con la noción de desarrollo sostenible, la Agenda 2030 para el Desarrollo Sostenible, a diferencia de otras agendas globales de desarrollo, incorpora metas específicas asociadas con la migración, entre ellas la meta 10.7 que propone facilitar la migración, la cual sigue más o menos el mismo lenguaje y contenidos del Pacto, con el agregado de la propuesta de políticas migratorias planificadas y bien gestionadas. Al menos 6 de los 17 ODS establecen metas directamente relacionadas con la migración internacional; 3 de éstas (metas 5.2, 8.7 y 16.2) fijan como objetivo para 2030 el poner fin con la trata y tráfico de personas, mientras que otras metas buscan garantizar los derechos laborales de los migrantes en los países de destino (8.8), la reducción de los costos de transacción de las remesas (10.c), la disponibilidad de becas para estudiar en el extranjero (4.b), la retención de los 
trabajadores de la salud en los países en desarrollo (3.c) y proporcionar acceso a identificación legal y registro de nacimiento (16.9).

En síntesis, la visión desde las Naciones Unidas es claramente afirmativa respecto de la migración internacional y se funda en la protección de los derechos humanos. Si existe una nueva iniciativa como el Pacto analizado es porque las que le preceden han sido insuficientes para lograr objetivos de protección de derechos.

\section{CUADRO 1}

Metas de la Agenda 2030 para el Desarrollo Sostenible referentes a la migración internacional

ODS

Meta asociada a la migración internacional

\section{2}

3 sulo

3 VEeEkshar $-10$

4 Euccaion

पأ

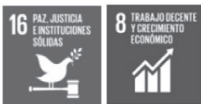

5 minum

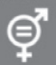

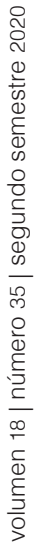

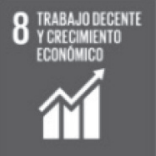

10 REDUCEION DELAS DESIEUAIDADES $\rightarrow$ 2

16 paz. Uusticia

16 EALSIIIUCIONES sólIDAS $\sum_{12}^{2}$

3.c Aumentar la capacitación y la retención del personal de salud en los países en desarrollo.

4.b Aumentar el número de becas disponibles para estudiar en el extranjero.

5.2/ 8.7/ 16.2 Erradicar la trata de personas y el tráfico ilícito de migrantes.

17 Munzusperea LOGRaR
LOS Oavintivos $\$$

17.18 Aumentar la disponibilidad de datos desglosados por condición migratoria. 


\section{Principios y objetivos del Pacto}

El Pacto se define con 10 principios relacionados con distintos ejes, por ejemplo, con la perspectiva de género, los derechos humanos, el desarrollo sostenible, el estado de derecho y el debido proceso y, quizá entre lo más relevante, con el interés superior del niño. Dicho conjunto de principios es un acervo de valor que tiene este instrumento, aun cuando falta una evaluación de su implementación.

Al mismo tiempo, el Pacto contiene los principios de soberanía nacional y otros enfoques de gobierno, que también fueron incluidos durante las negociaciones. Todos esos principios están entrecruzados, lo que da lugar a evidentes tensiones, por ejemplo, entre la soberanía nacional y el interés superior del niño. En este ejemplo, surge la inquietud sobre si cabe suponer que la soberanía nacional queda supeditada al interés superior del niño, como debería suceder, o si hay excepciones. Evidentemente, hace falta debatir más acerca del tema. Al respecto, es importante destacar que el párrafo 56 de la citada Declaración establece que «los niños no deben ser penalizados ni sometidos a medidas punitivas por su estatus migratorio ni por el de sus padres» (ONU, 2016:14).

FIGURA 1

Principios guía del Pacto

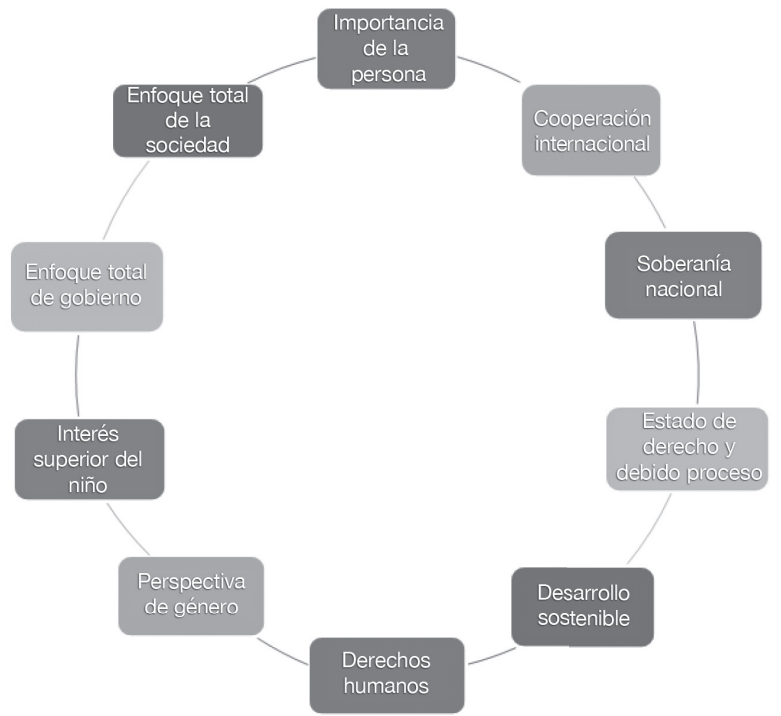




\section{CUADRO 2}

Objetivos para la migración segura, ordenada y regular

Objetivos

1. Recopilar y utilizar datos exactos y desglosados para formular políticas con base empírica.

2. Minimizar los factores adversos y estructurales que obligan a las personas a abandonar su país de origen.

3. Proporcionar información exacta y oportuna en todas las etapas de la migración.

4. Velar por que todos los migrantes tengan pruebas de su identidad jurídica y documentación adecuada.

5. Aumentar la disponibilidad y flexibilidad de las vías de migración regular.

6. Facilitar la contratación equitativa y ética y salvaguardar las condiciones que garantizan el trabajo decente.

7. Abordar y reducir las vulnerabilidades en la migración.

8. Salvar vidas y emprender iniciativas internacionales coordinadas sobre los migrantes desaparecidos.

9. Reforzar la respuesta transnacional al tráfico ilícito de migrantes.

10. Prevenir, combatir y erradicar la trata de personas en el contexto de la migración internacional.

11. Gestionar las fronteras de manera integrada, segura y coordinada.

12. Aumentar la certidumbre y previsibilidad de los procedimientos migratorios para la adecuada verificación de antecedentes, evaluación y derivación.

13. Utilizar la detención de migrantes sólo como último recurso y buscar otras alternativas.

14. Mejorar la protección, asistencia y cooperación consulares a lo largo de todo el ciclo migratorio.

15. Proporcionar a los migrantes acceso a servicios básicos.

16. Empoderar a los migrantes y las sociedades para lograr la plena inclusión y la cohesión social.

17. Eliminar todas las formas de discriminación y promover un discurso público con base empírica para modificar las percepciones de la migración.

18. Invertir en el desarrollo de aptitudes y facilitar el reconocimiento mutuo de aptitudes, cualificaciones y competencias.

19. Crear las condiciones necesarias para que los migrantes y las diásporas puedan contribuir plenamente al desarrollo sostenible en todos los países.

20. Promover transferencias de remesas más rápidas, seguras y económicas y fomentar la inclusión financiera de los migrantes.

21. Colaborar para facilitar el regreso y la readmisión en condiciones de seguridad y dignidad, así como la reintegración sostenible.

22. Establecer mecanismos para la portabilidad de la seguridad social y las prestaciones adquiridas.

23. Fortalecer la cooperación internacional y las alianzas mundiales para la migración segura, ordenada y regular. 


\section{CUADRO 3}

El Pacto Mundial para la Migración Segura, Ordenada y Regular según grandes áreas de política

\begin{tabular}{|c|c|c|c|c|}
\hline $\begin{array}{l}\text { Información } \\
y \text { cooperación }\end{array}$ & $\begin{array}{l}\text { Acceso a protección } \\
\text { social y a servicios }\end{array}$ & Regularización & $\begin{array}{l}\text { Soberanía } \\
\text { y seguridad de las } \\
\text { personas migrantes }\end{array}$ & Inclusión social \\
\hline $\begin{array}{l}\text { 1. Recolectary } \\
\text { utilizar datos } \\
\text { precisos y } \\
\text { desagregados } \\
\text { como base para } \\
\text { políticas basadas } \\
\text { en evidencia. }\end{array}$ & $\begin{array}{l}\text { 4. Proveer a todos } \\
\text { los migrantes de } \\
\text { pruebas de identidad } \\
\text { legal, identificación } \\
\text { adecuada y } \\
\text { documentación. }\end{array}$ & $\begin{array}{l}\text { 2. Minimizar los } \\
\text { factores adversos } \\
\text { y estructurales } \\
\text { que compelen } \\
\text { a las personas a } \\
\text { dejar su país de } \\
\text { origen. }\end{array}$ & $\begin{array}{l}\text { 8. Salvar vidas } \\
\text { y establecer } \\
\text { esfuerzos } \\
\text { internacionales } \\
\text { coordinados } \\
\text { para migrantes } \\
\text { desaparecidos. }\end{array}$ & $\begin{array}{l}\text { 16. Empoderar } \\
\text { a migrantes } \\
\text { y sociedades } \\
\text { para lograr una } \\
\text { inclusión y } \\
\text { cohesión social } \\
\text { integral. }\end{array}$ \\
\hline $\begin{array}{l}\text { 3. Proveer } \\
\text { información } \\
\text { adecuada y } \\
\text { oportuna en } \\
\text { todas las etapas } \\
\text { de la migración. }\end{array}$ & $\begin{array}{l}\text { 14. Extender } \\
\text { protección } \\
\text { consular, asistencia } \\
\text { y cooperación a } \\
\text { lo largo del ciclo } \\
\text { migratorio. }\end{array}$ & $\begin{array}{l}\text { 5. Mejorar la } \\
\text { disponibilidad } \\
\text { y flexibilidad } \\
\text { de los caminos } \\
\text { de la migración } \\
\text { regular. }\end{array}$ & $\begin{array}{l}\text { 9. Fortalecer } \\
\text { la respuesta } \\
\text { transnacional } \\
\text { al tráfico de } \\
\text { migrantes. }\end{array}$ & $\begin{array}{l}\text { 17. Eliminar } \\
\text { todas las formas } \\
\text { de discriminación } \\
\text { y promover un } \\
\text { discurso público } \\
\text { basado en hechos } \\
\text { para moldear la } \\
\text { percepción de la } \\
\text { migración. }\end{array}$ \\
\hline \multirow[t]{3}{*}{$\begin{array}{l}\text { 23. Fortalecer } \\
\text { la cooperación } \\
\text { internacional } \\
\text { y las alianzas } \\
\text { globales para } \\
\text { una migración } \\
\text { segura, ordenada } \\
\text { y regular. }\end{array}$} & $\begin{array}{l}\text { 15. Proveer acceso } \\
\text { a servicios básicos } \\
\text { para migrantes. }\end{array}$ & $\begin{array}{l}\text { 6. Facilitar } \\
\text { reclutamiento } \\
\text { justo, ético y } \\
\text { las condiciones } \\
\text { que aseguren el } \\
\text { trabajo decente. }\end{array}$ & $\begin{array}{l}\text { 10. Prevenir y } \\
\text { combatir la trata } \\
\text { de personas } \\
\text { en el contexto } \\
\text { de migración } \\
\text { internacional. }\end{array}$ & $\begin{array}{l}\text { 18. Invertir en } \\
\text { el desarrollo } \\
\text { de habilidades } \\
\text { y facilitar el } \\
\text { reconocimiento } \\
\text { de éstas, las } \\
\text { calificaciones y } \\
\text { competencias. }\end{array}$ \\
\hline & $\begin{array}{l}\text { 20. Promover las } \\
\text { transferencias de } \\
\text { remesas de forma } \\
\text { más rápida, barata y } \\
\text { segura, y contribuir } \\
\text { a la inclusión } \\
\text { financiera de los } \\
\text { migrantes. }\end{array}$ & $\begin{array}{l}\text { 7. Abordar } \\
\text { y reducir las } \\
\text { vulnerabilidades } \\
\text { en la migración. }\end{array}$ & $\begin{array}{l}\text { 11. Gestionar } \\
\text { las fronteras } \\
\text { de una forma } \\
\text { integrada, segura } \\
\text { y coordinada. }\end{array}$ & $\begin{array}{l}\text { 19. Crear } \\
\text { condiciones para } \\
\text { la contribución } \\
\text { de migrantes } \\
\text { y diásporas } \\
\text { al desarrollo } \\
\text { sostenible en } \\
\text { todos los países. }\end{array}$ \\
\hline & $\begin{array}{l}\text { 22. Establecer } \\
\text { mecanismos para } \\
\text { la portabilidad de } \\
\text { los beneficios de } \\
\text { seguridad social y } \\
\text { beneficios ganados. }\end{array}$ & $\begin{array}{l}\text { 12. Fortalecer } \\
\text { la certeza y las } \\
\text { capacidades de } \\
\text { anticipación } \\
\text { en los procesos } \\
\text { migratorios. }\end{array}$ & $\begin{array}{l}\text { 13. Utilizar la } \\
\text { detención de } \\
\text { migrantes sólo } \\
\text { como una medida } \\
\text { de último recurso } \\
\text { y trabajar hacia } \\
\text { alternativas. }\end{array}$ & $\begin{array}{l}\text { 21. Cooperar en } \\
\text { la facilitación } \\
\text { del retorno } \\
\text { sustentable, la } \\
\text { readmisión y la } \\
\text { reintegración. }\end{array}$ \\
\hline
\end{tabular}

Fuente: elaboración propia con base en Global Compact for Migration, 2018, y en Maldonado, Martínez y Martínez, 2018. 
Las líneas de acción (variables en número y naturaleza) que posee cada uno de los 23 objetivos del Pacto derivan de los grandes temas identificados en los precedentes indicados y a partir de un largo trabajo realizado con la participación de los Estados miembros de las Naciones Unidas. En primer lugar, en términos de política pública, el objetivo 1 está centrado en recolectar y utilizar datos precisos y desagregados para diseñar y evaluar políticas basadas en evidencia. Es un objetivo elemental, pero a la vez es convocante, dado que se ha observado cómo algunos países que no se incorporaron al proceso del Pacto han tomado conciencia del beneficio que puede significarles participar de proyectos globales de recopilación de información, de intercambio y de manejo de datos de distinta naturaleza relativos a los procesos migratorios. Lo mismo se puede señalar respecto del objetivo 3, que establece la provisión de información adecuada y oportuna en todas las etapas de la migración, y del objetivo 23, que plantea fortalecer la cooperación internacional y las alianzas globales para una migración segura, ordenada y regular.

Hasta el momento, es claro que existe un alto interés en el objetivo 1, porque se considera muy positivo beneficiarse de las diversas iniciativas globales y regionales que están por consolidarse. En segundo lugar, concerniente al tema de acceso a protección social y servicios, se puede destacar, por ejemplo, el objetivo 20 de promover las transferencias de remesas de forma más rápida, barata y segura, y contribuir asimismo a la inclusión financiera de las personas migrantes. Existen diversas dimensiones vinculadas a la inclusión social que pueden mencionarse, como el acceso a servicios sociales, plasmado en el objetivo 15 de proveer acceso a los servicios básicos para los migrantes. En ese marco, también es relevante el objetivo 22 que plantea el establecimiento de mecanismos para la portabilidad de los beneficios de seguridad social y beneficios ganados, como la portabilidad de pensiones.

Este tipo de dispositivos, propuestas y contenidos, que incluyen asuntos como la protección, asistencia consular y el acceso a servicios básicos para migrantes, eran impensados hace 10 años en iniciativas globales, lo que demuestra que existe un interés deliberado por incluir temas de carácter social en la gestión de la migración y en la gobernanza migratoria.

En tercer lugar, el tema de la regularización ha sido definido como uno de los grandes imperativos en todas las regiones, aunque con lecturas distintas, reflejado en los objetivos 2, 5, 6, 7y 12. Es claro que el abordaje del tema fue uno de los principales obstáculos en las negociaciones, por todo lo que variadamente 
significa el manejo de la migración irregular para los Estados. Otro de los temas centrales del pacto es el de la soberanía y seguridad para las personas migrantes, abordado en los objetivos 8, 9, 10, 11 y 13. Éstos incluyen la gestión de fronteras y la lucha contra la trata de personas y contra el tráfico de migrantes, así como el imperativo de salvar vidas y establecer esfuerzos internacionales coordinados para la búsqueda de migrantes desaparecidos, como lo estipula el objetivo 8.

Por desgracia, la desaparición de migrantes ha sido una constante en las últimas décadas, en especial en regiones como el Mediterráneo, entre otras localizaciones específicas. Y cercana a esa problemática, otra temática central, principalmente para la sociedad civil, ha sido plantear, a través del objetivo 13, la lucha contra la detención de migrantes (utilizar la detención de migrantes sólo como una medida de último recurso y trabajar hacia alternativas). Varios de esos objetivos son percibidos como una de las justificaciones éticas más importantes del Pacto, lo que contrasta fuertemente con los cuestionamientos realizados durante las negociaciones, por aquellos países que se retiraron y señalaron -y siguen señalando-, en cuanto a que este principio u objetivo se contrapone a la soberanía de los países.

Con relación a la inclusión social, hay varios aspectos interesantes. En primer lugar, el Pacto delinea para los próximos años una conjunción de propuestas sobre los grandes temas de interés y de estudio concernientes a la migración y numerosos aportes para el diseño de políticas públicas. Por ejemplo, el objetivo 17 plantea la necesidad de eliminar todas las formas de discriminación y promover un discurso público basado en evidencias que orienta una percepción de la migración.

Por su parte, el objetivo 16 de empoderar a migrantes y sociedades para una inclusión y cohesión social integral, se entrecruza con el tema de los reconocimientos de calificaciones y competencias (objetivo 18), que por años fue muy disperso en las iniciativas y ahora está consolidado en un documento con ese carácter. No menos importante es lo que tiene que ver con la facilitación del retorno sustentable, la readmisión y la reintegración (objetivo 21). Hay que considerar que en la región, así como en otras, el tema del retorno es central para la política pública contemporánea, adicionalmente a todos los otros procesos que acompañan a la migración, como sucede en el norte de Centroamérica o como se advirtió en 2020 con los impactos de la pandemia sobre algunas familias e individuos que perdieron trabajo e ingresos y vieron en el retorno una salida a sus dificultades. 
También es relevante que el Pacto aborde temas como el racismo y la xenofobia, y se hace referencia a ellos y se recogen, además, algunas iniciativas previas; es el caso del principio guía de derechos humanos, en el cual los Estados se comprometen a eliminar todas las formas de discriminación, incluyendo el racismo, la xenofobia y la intolerancia hacia los migrantes y sus familias. Precisamente, tal como se mencionó anteriormente, el objetivo 17 plantea eliminar todas las formas de discriminación y promover un discurso público basado en evidencia para orientar y moldear la percepción acerca de la migración, al condenar actos y manifestaciones de racismo, discriminación racial, violencia y xenofobia contra los migrantes, y al involucrar a toda la comunidad.

\section{Cruciales indefiniciones}

La promoción de acciones en cada uno de los objetivos del Pacto asemeja a los planes de acción emanados de instrumentos internacionales como la Conferencia Internacional sobre Población y Desarrollo de 1994. Esta instancia tuvo seguimiento general y dio origen, en 2013, a la adopción del Consenso de Montevideo sobre Población y Desarrollo de América Latina y el Caribe, con sus nueve capítulos y medidas prioritarias en cada uno de ellos (Cepal, 2013). ¿Constituyen entonces las líneas de acción del Pacto un plan de acción acomodado a las circunstancias de un tema controversial donde muchos Estados no aceptan injerencias como lo ha sido la migración en las últimas décadas? Claramente se trata apenas de una aproximación que con seguridad limitará las posibilidades de implementación.

Más allá de dicha indefinición, nuestra aprensión mayor es que la misma fortaleza del Pacto anclada en la promoción de una gobernanza integral de la migración y la adopción de políticas afines a varios principios indiscutiblemente relevantes, se vuelve su principal limitación. En efecto, un análisis de las acciones identificadas en muchos de los objetivos muestra que no existen definiciones claras acerca de indicadores, fuentes, metadatos, horizontes y metas. Ello puede llevar, además, a la libre interpretación del proceso migratorio ordenado, seguro y regular.

Sólo como ejemplo, el objetivo 1 enumera 11 acciones de distinto orden destinadas a fortalecer la evidencia de los procesos migratorios, en lo que constituye una antigua tradición en las Naciones Unidas. Difícilmente se puede estar en desacuerdo con propuestas de mejoramiento de estadísticas y fortalecimiento de la coordinación intersectorial nacional, o el apoyo a centros regionales de 
investigación y formación u observatorios sobre la migración, o la difusión de datos provenientes de distintas fuentes de información. ¿Pero con qué recursos se puede apoyar a los países más necesitados? ¿Qué plazos cabe darse para alcanzar alguna meta por ahora indefinida en cuanto al mejoramiento de la evidencia? El objetivo 1 cierra con la siguiente acción propuesta: «k) Cooperar con los interesados pertinentes en los países de origen, tránsito y destino para aumentar las investigaciones, estudios y encuestas sobre la interrelación entre la migración y las tres dimensiones del desarrollo sostenible, las contribuciones y aptitudes de los migrantes y las diásporas, y sus vínculos con los países de origen y destino» (Asamblea General de las Naciones Unidas, 2018:9).

\section{¿Hacia dónde vamos?}

En perspectiva, el proceso de implementación del Pacto es promisorio por las evidentes fortalezas que resaltan de la integralidad de sus contenidos desplegados en su lenguaje y sus objetivos, a la vez que es susceptible de cuestionamientos sobre su implementación y sus propuestas. Al mismo tiempo, parece un acertado ejemplo de las posibilidades de soft law para encarar las profundas injusticias que enfrentan cotidianamente muchas personas migrantes, pues motiva una amplia participación de organizaciones, instituciones y de los propios Estados.

Para finalizar, se pueden mencionar varias amenazas, independientes de la implementación del Pacto. Por una parte, en distintos medios se sigue hablando de migración segura, ordenada y regular con la muy probable diferencial interpretación al respecto, desde no tener claridad en los alcances que ello implica (principios, objetivos y acciones), hasta asumirlo como un sometimiento para reforzar el control migratorio, las deportaciones, las detenciones, como un elemento estratégico de la gestión migratoria contemporánea. Todo esto será un tema que se decantará lentamente. A su vez, las relaciones con el pacto sobre refugiados tampoco se han establecido con claridad, a pesar del interés de algunos gobiernos por relevar las sinergias que tienen ambos pactos. Por otra parte, la gobernanza de la migración es un tema altamente complejo y que se debe discutir, en particular cuando se dan confusiones entre refugiados y migrantes. No es sólo la dimensión política. Adicionalmente, el retiro de Estados Unidos desde los prolegómenos del pacto, al que le siguieron después otros países, fue algo muy complejo en su momento, en especial para la región que concentra su emigración en ese país. 


\section{Referencias}

Asamblea General de las Naciones Unidas (2018), "Proyecto de documento final de la Conferencia Intergubernamental encargada de Aprobar el Pacto Mundial para la Migración Segura, Ordenada y Regular», Marrakech, Marruecos, 10 y 11 de diciembre de 2018, en http://undocs.org/es/A/CONF.231/3

Comisión Económica para América Latina y el Caribe (Cepal) (2013), Consenso de Montevideo sobre Población y Desarrollo, Primera reunión de la Conferencia Regional sobre Población y Desarrollo de América Latina y el Caribe, Cepal, en http://www. cepal.org/es/publicaciones/21835-consenso-montevideo-poblacion-desarrollo

Global Compact for Migration (2018), «Global Compact for Safe, Orderly and Regular Migration. Draft Rev 2», 28 de mayo de 2018, en https:/childrenonthemove.org/ wp-content/uploads/2018/03/Child-Rights-Initiative-Language_Draft-Rev-2REVISED.pdf

Maldonado Valera, C., J. Martínez Pizarro y R. Martínez (2018), «Protección social y migración: una mirada desde las vulnerabilidades a lo largo del ciclo de la migración y de la vida de las personas», Documentos de Proyectos (LC/TS.2018/62), Santiago, Comisión Económica para América Latina y el Caribe (Cepal).

Martínez Pizarro, Jorge (2015), "Oportunidades e incertidumbre a la luz del Segundo Diálogo de Alto Nivel sobre Migración Internacional y Desarrollo», Coyuntura Demográfica (7), pp. 61-65.

(2017), «Migración internacional en Chile: la necesidad de genuinos debates», Palabra Pública, 4, pp. 42-43.

Organización de las Naciones Unidas (ONU) (2015), Transformar nuestro mundo: la Agenda 2030 para el Desarrollo Sostenible, en https://documents-dds-ny.un.org/doc/UNDOC/ GEN/N15/291/93/PDF/N1529193.pdf?OpenElement

(2016), Declaración de Nueva York para los Refugiados y los Migrantes. (2018), Pacto Mundial sobre los Refugiados, Nueva York, en https://acnur. org/5c782d124

Ramírez Jacques, Iréri Ceja y Yolanda Alfaro (2019), «La Conferencia Sudamericana de Migraciones y el Proceso Puebla: ¿entre la seguridad y los derechos?», Periplos, Revista de Investigación sobre Migración, 3(1).

United Nations (2015), Integrating migration into the 2030 Agenda for Sustainable Development. Population Facts, 5, pp. 1-4. 